\title{
Performance of Alamouti Transmit Diversity Over Time-Varying Rayleigh-Fading Channels
}

\author{
Antony Vielmon, Ye (Geoffrey) Li, and John R. Barry
}

\begin{abstract}
We analyze the impact of a time-varying Rayleighfading channel on the performance of an Alamouti transmit-diversity scheme. We propose several optimal and suboptimal detection strategies for mitigating the effects of a time-varying channel, and derive expressions for their bit-error probability as a function of the channel correlation coefficient $\rho$. We find that the maximum-likelihood detector that optimally compensates for the timevarying channel is very tolerant to time-varying fading, attaining full diversity order even for the extreme case of $\rho=0$. In contrast, although lower in complexity, the suboptimal schemes suffer a diversity penalty and are thus suitable only for slowly fading channels.
\end{abstract}

Index Terms-Alamouti's approach, performance analysis, Rayleigh-fading channels, time-varying channels, transmit diversity.

\section{INTRODUCTION}

$\mathbf{T}$ RANSMIT diversity has emerged in the last decade as an effective means for achieving spatial diversity in fading channels with an antenna array at the transmitter. In the design and analysis of such schemes, it is generally assumed that the channel is static for the duration of one space-time codeword. In this letter, we investigate the impact of a time-varying channel on the performance of the transmit-diversity scheme proposed by Alamouti [1]. We propose various detection strategies that take into account the time-varying nature of the channel and assess their performance through analysis and simulation.

\section{Channel Model and Assumptions}

A transmitter with two antennas employing the transmit-diversity scheme of Alamouti [1] requires two signaling periods to convey a pair of finite-alphabet symbols $x_{1}$ and $x_{2}$; during the first symbol period, the symbols transmitted from antenna one and antenna two, respectively, are $x_{1}$ and $x_{2}$, and during the second symbol period they are $-x_{2}^{*}$ and $x_{1}^{*}$. Consider a receiver with one antenna, and assume a flat-fading channel model. Let $h_{1}$ and $h_{2}$ denote the equivalent complex channel coefficients between the two transmit antennas and the receiver antenna during the first symbol period, and let $\hat{h}_{1}$ and $\hat{h}_{2}$ denote the coefficients during the second period, so that the receiver observations $r_{1}$ and $r_{2}$ corresponding to the two symbol periods are given by

$$
\begin{aligned}
& r_{1}=h_{1} x_{1}+h_{2} x_{2}, \\
& r_{2}=-\tilde{h}_{1} x_{2}^{*}+\tilde{h}_{2} x_{1}^{*} .
\end{aligned}
$$

Manuscript received March 6, 2002; revised March 7, 2003; accepted May 7,2003 . The editor coordinating the review of this paper and approving it for publication is P. F. Driessen. This work was supported in part by The National Science Foundation under Grant CCR-0082329 and Grant CCR-0121565.

The authors are with the School of Electrical and Computer Engineering, Georgia Institute of Technology, Atlanta, GA 30332-0250 USA (e-mail: liye@ece.gatech.edu).

Digital Object Identifier 10.1109/TWC.2004.833524
Equivalently, by conjugating $r_{2}$, the following convenient matrix representation results:

$$
\left[\begin{array}{l}
r_{1} \\
r_{2}^{*}
\end{array}\right]=\left[\begin{array}{cc}
h_{1} & h_{2} \\
\tilde{h}_{2}^{*} & -\tilde{h}_{1}^{*}
\end{array}\right]\left[\begin{array}{l}
x_{1} \\
x_{2}
\end{array}\right]+\left[\begin{array}{c}
w_{1} \\
w_{2}^{*}
\end{array}\right]
$$

or with obvious notation

$$
\boldsymbol{r}=\mathbf{H} \boldsymbol{x}+\boldsymbol{w}
$$

where $\boldsymbol{w}$ represents noise.

In this letter, we make the following assumptions about the channel model (3):

1) white Gaussian noise, so that $\boldsymbol{w}$ is a zero-mean circularly symmetric complex Gaussian random vector satisfying $E\left[\boldsymbol{w} w^{*}\right]=N_{0} \mathbf{I}$

2) spatially symmetric Rayleigh fading, so that $h_{1}, \tilde{h}_{1}, h_{2}$, and $\tilde{h}_{2}$ are identically distributed, zero-mean unit-variance circularly symmetric complex jointly Gaussian random variables satisfying $E\left[\left|h_{1}\right|^{2}\right]=E\left[\left|\tilde{h}_{1}\right|^{2}\right]=E\left[\left|h_{2}\right|^{2}\right]=$ $E\left[\left|\tilde{h}_{2}\right|^{2}\right]=1$

3) sufficient antenna spacing, so that the pair $\left(h_{1}, \tilde{h}_{1}\right)$ is independent of the pair $\left(h_{2}, \tilde{h}_{2}\right)$; relaxing this constraint would be possible, but it would complicate the analysis and it would detract from our main aim of studying the impact of time variations;

4) temporally symmetric Rayleigh fading, so that the correlation $\rho$ between $h_{1}$ and $\tilde{h}_{1}$ is the same as that between $h_{2}$ and $\tilde{h}_{2}$, namely $E\left[h_{1} \tilde{h}_{1}^{*}\right]=E\left[h_{2} \tilde{h}_{2}^{*}\right]=\rho$;

5) perfect knowledge of $h_{1}, \tilde{h}_{1}, h_{2}$, and $\tilde{h}_{2}$ at the receiver;

6) binary phase-shift keying modulation with $x_{i} \in$ $\{ \pm \sqrt{E / 2}\}$, where $E$ is the average received energy per bit, so that the average received signal-to-noise ratio (SNR) per bit is $E / N_{0}$.

The key parameter in our model is the correlation parameter $\rho$, which will be near unity for slowly fading channels, but which could be small in rapidly varying channels. For example, when the maximum Doppler frequency using Jake's channel model is $10 \%$ of the baud rate, the correlation is $\rho=J_{0}(2 \pi \times 0.1) \approx 0.90$, where $J_{0}$ is the zeroth-order Bessel function of the first kind [2]. On the other hand, when $h_{i}$ and $\tilde{h}_{i}$ represent the channel frequency responses of two adjacent tones in orthogonal frequency-division multiplexing, $\rho$ is determined by the channel delay profile.

\section{STRATEGIES FOR ReCEIVER DESIGN}

In this section, we propose three methods for detecting an Alamouti space-time code when the channel is time-varying. The first is the joint maximum-likelihood (ML), the second is 
the decision-feedback (DF) detector, and the third is a zeroforcing $(\mathrm{ZF})$ linear detector.

\section{A. ML Detector}

Because of the white Gaussian noise, the joint ML detector chooses the pair of symbols $\boldsymbol{x}$ to minimize

$$
\|\boldsymbol{r}-\mathbf{H} \boldsymbol{x}\|^{2} .
$$

Let $\mathbf{R}=\mathbf{H}^{*} \mathbf{H}$ denote the cascade of $\mathbf{H}$ with its matched filter; since $\mathbf{R}$ is Hermitian, it possesses a unique Cholesky factorization of the form $\mathbf{R}=\mathbf{G}^{*} \mathbf{G}$, where $\mathbf{G}$ is lower triangular with real diagonal elements. With $\mathbf{H}$ defined by (2), it is easily verified that

$$
\mathbf{G}=\frac{1}{\sqrt{\left|\tilde{h}_{1}\right|^{2}+\left|h_{2}\right|^{2}}}\left[\begin{array}{cc}
\left|h_{1} \tilde{h}_{1}^{*}+h_{2} \tilde{h}_{2}^{*}\right| & 0 \\
h_{1} h_{2}^{*}-\tilde{h}_{1} \tilde{h}_{2}^{*} & \left|\tilde{h}_{1}\right|^{2}+\left|h_{2}\right|^{2}
\end{array}\right] .
$$

Multiplying both $\boldsymbol{r}$ and $\mathbf{H} \boldsymbol{x}$ in (4) by the unitary matrix $\mathbf{G}^{-*} \mathbf{H}^{*}$, we find that the ML detector can equivalently choose $\boldsymbol{x}$ to minimize

$$
\|z-\mathbf{G} x\|^{2}
$$

where we have introduced

$$
z=\mathbf{G}^{-*} \mathbf{H}^{*} \boldsymbol{r}
$$

The linear combiner of (7) represents the whitened-matched filter (WMF) for the matrix channel (3). Substituting (3), we find that the output $\boldsymbol{z}$ of the WMF is related to $\boldsymbol{x}$ by

$$
z=\mathbf{G} \boldsymbol{x}+\boldsymbol{n}
$$

where the white Gaussian noise $\boldsymbol{n}$ has the same statistics as $\boldsymbol{w}$.

\section{B. DF Detector}

The DF detector uses a decision about $x_{1}$ to help make a decision about $x_{2}$ [9]. It builds on the WMF output. In particular, because the WMF channel model $\mathbf{G}$ is lower triangular, there is no crosstalk from $x_{2}$ to $z_{1}$, and thus a suboptimal decision $\hat{x}_{1}$ regarding $x_{1}$ can be found by quantizing $z_{1}$, ignoring $z_{2}$. Then, assuming this decision is correct, the contribution from $x_{1}$ in $z_{2}$ can be recreated and subtracted off, allowing the receiver to determine the decision by quantizing the resulting difference $D$, where

$$
D=z_{2}-\frac{h_{1} h_{2}^{*}-\tilde{h}_{1} \tilde{h}_{2}^{*}}{\sqrt{\left|\tilde{h}_{1}\right|^{2}+\left|h_{2}\right|^{2}}} \cdot \hat{x}_{1} .
$$

\section{ZF Linear Detector}

A linear detector computes the following:

$$
\boldsymbol{y}=\mathrm{Cr}
$$

then makes a decision about $x_{i}$ based solely on $y_{i}$, for $i=1,2$. A zero-forcing linear detector chooses $\mathbf{C}$ so as to force the crosstalk to zero, so that the cascade $\mathbf{C H}$ is a real nonnegative diagonal matrix. Since $\mathbf{H}$ is square and full rank with proba- bility one, the ZF detector is clearly of the form $\mathbf{C}=\mathbf{A H}^{-1}$ for some real nonnegative diagonal matrix $\mathbf{A}$. We can make the detector unique by adding the additional constraint that the combiner does not change the noise variance, so that the noise components in $\boldsymbol{y}$ have second moment $N_{0}$, the same as those in $\boldsymbol{r}$. In other words, because the autocorrelation matrix of the noise after a combiner of the form $\mathbf{C}=\mathbf{A} \mathbf{H}^{-1}$ is $N_{0} \mathbf{A} \mathbf{R}^{-1} \mathbf{A}$, we choose $\mathbf{A}$ so that $\mathbf{A} \mathbf{R}^{-1} \mathbf{A}$ has ones on the diagonal. It is easily verified that the solution leads to the following $\mathrm{ZF}$ combiner:

$$
\begin{aligned}
\mathbf{C}= & \frac{h_{1} \tilde{h}_{1}^{*}+h_{2} \tilde{h}_{2}^{*} \mid}{h_{1} \tilde{h}_{1}^{*}+h_{2} \tilde{h}_{2}^{*}} \\
& \times\left[\begin{array}{cc}
\left(\left|\tilde{h}_{1}\right|^{2}+\left|h_{2}\right|^{2}\right)^{-1 / 2} & 0 \\
0 & \left(\left|h_{1}\right|^{2}+\left|\tilde{h}_{2}\right|^{2}\right)^{-1 / 2}
\end{array}\right] \\
& \times\left[\begin{array}{cc}
\tilde{h}_{1}^{*} & h_{2} \\
\tilde{h}_{2}^{*} & -h_{1}
\end{array}\right] .
\end{aligned}
$$

Substituting (11) and (3) into (10) yields

$$
\begin{aligned}
\boldsymbol{y} & =\left|h_{1} \tilde{h}_{1}^{*}+h_{2} \tilde{h}_{2}^{*}\right| \\
& \times\left[\begin{array}{cc}
\left(\left|\tilde{h}_{1}\right|^{2}+\left|h_{2}\right|^{2}\right)^{-1 / 2} & 0 \\
0 & \left(\left|h_{1}\right|^{2}+\left|\tilde{h}_{2}\right|^{2}\right)^{-1 / 2}
\end{array}\right] \boldsymbol{x}+\tilde{\boldsymbol{n}}
\end{aligned}
$$

where the noise components $\tilde{n}_{1}$ and $\tilde{n}_{2}$ are identically distributed, each being zero-mean complex Gaussian with $E\left[\left|\tilde{n}_{i}\right|^{2}\right]=N_{0}$. Although $\tilde{n}_{1}$ and $\tilde{n}_{2}$ are correlated, the ZF detector ignores the correlation, and arrives at suboptimal decisions by independently quantizing $y_{1}$ and $y_{2}$.

Comparing (12) to (8), we see that the first output $y_{1}$ of the ZF detector is identical to the first output $z_{1}$ of the WMF. (The second outputs differ, however.)

\section{All Detectors Are Equivalent if the Channel Is Static}

The ML, DF, and ZF detectors operate in distinct ways, and the performance difference between them can be significant. However, it is worth emphasizing at this stage that the ZF, DF, and ML detectors all converge to the same detector in the special case of a static channel.

Consider first the ML detector. If the channel is static, so that $\tilde{h}_{1}=h_{1}$ and $\tilde{h}_{2}=h_{2}$, then $\mathbf{G}$ of (5) reduces to the diagonal matrix $\sqrt{\left|h_{1}\right|^{2}+\left|h_{2}\right|^{2}} \mathbf{I}$, and (8) reduces to

$$
\boldsymbol{z}=\sqrt{\left|h_{1}\right|^{2}+\left|h_{2}\right|^{2}} \boldsymbol{x}+\boldsymbol{n} .
$$

The joint ML detector thus reduces to a pair of independent scalar detectors, significantly reducing complexity. Indeed, the desire to diagonalize the channel using a MF was what lead to the Alamouti transmit-diversity scheme in the first place. Clearly, because there is no crosstalk after the WMF, the coefficient of $\hat{x}_{1}$ in (9) reduces to zero and, thus, the DF reduces to the ML detector for the static case.

Finally, the ZF detector of (12) also reduces to (13) when the channel is static.

\section{Performance Analysis-Special Cases}

Before considering the general case of arbitrary correlation, it will be instructive to focus first on two extreme cases: the fully 
correlated or static channel, where $\rho=1$, and the uncorrelated channel, where $\rho=0$. In the remainder of this section, we derive expressions for the bit-error probability for these extreme cases, assuming the ZF linear detector of (11). We assume uncoded binary phase-shift keying modulation. We remark that, from the symmetry of the channel model, we only need to derive the error probability for the first symbol $x_{1}$, knowing that the second symbol will have the same error probability.

\section{A. Fully Correlated $(\rho=1)$ Static Channel}

The analysis for the static case is well-known [2]. The first component in (12) is

$$
y_{1}=\sqrt{\left|h_{1}\right|^{2}+\left|h_{2}\right|^{2}} x_{1}+\tilde{n}_{1}
$$

so that the decision $\hat{x}_{1}=\operatorname{sign}\left(z_{1}\right)$ has bit-error probability $Q(\sqrt{2 \gamma})$, where $\gamma=\left(\left|h_{1}\right|^{2}+\left|h_{2}\right|^{2}\right) E /\left(2 N_{0}\right)$ is the instantaneous SNR per bit. The average SNR per bit is $E[\gamma]=E / N_{0}$. Since $h_{1}$ and $h_{2}$ are independent and Gaussian, $\gamma$ has a central chi-square distribution with four degrees of freedom, and probability density function (pdf)

$$
p(\gamma)=\frac{\gamma}{\left(E / 2 N_{0}\right)^{2}} \exp \left(-\gamma 2 N_{0} / E\right)
$$

Averaging $Q(\sqrt{2 \gamma})$ over this distribution yields [2]

$$
P_{e}=\frac{1}{4}\left(1-\frac{1}{\sqrt{1+\frac{2}{E / N_{0}}}}\right)^{2}\left(2+\frac{1}{\sqrt{1+\frac{2}{E / N_{0}}}}\right)
$$

\section{B. Uncorrelated Case $(\rho=0)$}

From (12), the first output of the ZF detector is

$$
y_{1}=\frac{\left|h_{1} \tilde{h}_{1}^{*}+h_{2} \tilde{h}_{2}^{*}\right|}{\sqrt{\left|\tilde{h}_{1}\right|^{2}+\left|h_{2}\right|^{2}}} x_{1}+\tilde{n} .
$$

The error probability is, thus, again of the form $Q(\sqrt{2 \mu})$, where $\mu$ is the effective instantaneous SNR for (17)

$$
\mu=\frac{\left|h_{1} \tilde{h}_{1}^{*}+h_{2} \tilde{h}_{2}^{*}\right|^{2}}{\left|\tilde{h}_{1}\right|^{2}+\left|h_{2}\right|^{2}} E /\left(2 N_{0}\right) .
$$

Introducing $u_{1}=\tilde{h}_{1} / \sqrt{\left|\tilde{h}_{1}\right|^{2}+\left|h_{2}\right|^{2}}$ and $u_{2}=$ $h_{2}^{*} / \sqrt{\left|\tilde{h}_{1}\right|^{2}+\left|h_{2}\right|^{2}}$, the effective SNR can equivalently be expressed as $\mu=|Y|^{2} E /\left(2 N_{0}\right)$, where $Y=u_{1}^{*} h_{1}+u_{2}^{*} \tilde{h}_{2}^{*}$ can be interpreted as a projection $\boldsymbol{u}^{*} \boldsymbol{h}$ of $\boldsymbol{h}=\left[h_{1}, \tilde{h}_{2}^{*}\right]^{T}$ in the direction of the independent unit vector $\boldsymbol{u}=\left[u_{1}, u_{2}\right]^{T}$. Since the pdf of $\boldsymbol{h}$ is symmetric, and since $\boldsymbol{h}$ is independent of $\boldsymbol{u}$, it follows that the pdf of $Y$ will be independent of $\boldsymbol{u}$, and choosing $\boldsymbol{u}=[1,0]^{T}$ we find that $Y$ has the same pdf as $h_{1}$. Thus, the effective SNR $\mu=|Y|^{2} E /\left(2 N_{0}\right)$ has mean value $E /\left(2 N_{0}\right)$, exactly half of what it was for the static channel, and it has a central chi-square distribution with two degrees of freedom

$$
p(\mu)=\frac{2}{E / N_{0}} \exp \left(-\mu 2 N_{0} / E\right) .
$$

Averaging $Q(\sqrt{2 \mu})$ over this distribution yields [2]

$$
P_{e}=\frac{1}{2}\left(1-\frac{1}{\sqrt{1+\frac{2}{E / N_{0}}}}\right)
$$

\section{Performance Analysis-General CASE}

\section{A. Performance of the ZF Linear Detector}

The effective instantaneous SNR at the first output of the ZF linear detector is given by (18). Let us introduce the random variables $\varepsilon_{1}$ and $\varepsilon_{2}$

$$
\begin{gathered}
\varepsilon_{1}=\frac{h_{1}-\rho \tilde{h}_{1}}{\sqrt{1-\rho^{2}}} \\
\varepsilon_{1}=\frac{\tilde{h}_{2}-\rho h_{2}}{\sqrt{1-\rho^{2}}}
\end{gathered}
$$

where we assume $|\rho|<1$. By construction, $\varepsilon_{1}$ and $\varepsilon_{2}$ are independent and identically distributed with the same pdf as $\tilde{h}_{1}$ and $h_{2}$, and furthermore, $\varepsilon_{1}$ is independent of $\tilde{h}_{1}$, and $\varepsilon_{2}$ is independent of $h_{2}$. Plugging (21) into (18) leads to

$\mu=\left|\rho \sqrt{\left|\tilde{h}_{1}\right|^{2}+\left|h_{2}\right|^{2}}+\sqrt{1-\rho^{2}} \frac{\tilde{h}_{1}^{*} \varepsilon_{1}+h_{2} \varepsilon_{2}^{*}}{\sqrt{\left|\tilde{h}_{1}\right|^{2}+\left|h_{2}\right|^{2}}}\right|^{2} E /\left(2 N_{0}\right)$.

To simplify this expression further, observe that the fraction $\left(t_{1}+j t_{2}\right)$ in (22) can be expressed as an inner product

$$
t_{1}+j t_{2}=\frac{\tilde{h}_{1}^{*} \varepsilon_{1}+h_{2} \varepsilon_{2}^{*}}{\sqrt{\left|\tilde{h}_{1}+\right|^{2}+\left|h_{2}\right|^{2}}}=(\boldsymbol{h} /\|\boldsymbol{h}\|)^{*} \boldsymbol{e}
$$

where $\boldsymbol{h}=\left[\tilde{h}_{1}, h_{2}^{*}\right]^{T}$ and $\boldsymbol{e}=\left[\varepsilon_{1}, \varepsilon_{2}^{*}\right]^{T}$. Since $\boldsymbol{e}$ is symmetric, the distribution of $t_{1}+j t_{2}$ reduces to the distribution of $h_{1}$, independent of $\boldsymbol{h}$ (and, thus, also independent of $\|\boldsymbol{h}\|$ ). Thus, (22) simplifies to

$$
\begin{aligned}
\mu & =\left|\rho \sqrt{\left|\tilde{h}_{1}\right|^{2}+\left|h_{2}\right|^{2}}+\sqrt{1-\rho^{2}}\left(t_{1}+j t_{2}\right)\right|^{2} E /\left(2 N_{0}\right) \\
& =\left(A+X_{1}\right)^{2}+X_{2}^{2}
\end{aligned}
$$


where we have introduced $A=\rho \sqrt{\left(\left.\tilde{h}_{1}\right|^{2}+\left|h_{2}\right|^{2}\right) E / 2 N_{0}}$ and $X_{i}=\sqrt{\left(1-\rho^{2}\right) E / 2 N_{0}} t_{i}$. Therefore, given $A, \mu$ has a noncentral chi-square distribution with two degrees of freedom, and pdf

$$
\begin{aligned}
P_{\mu \mid \mathrm{A}}(\mu \mid \alpha)= & \frac{2}{\left(1-\rho^{2}\right) E / 2 N_{0}} \exp \left(-2\left(a^{2}+\mu\right) /\left(\left(1-\rho^{2}\right)\right.\right. \\
& \left.\left.\times E / 2 N_{0}\right)\right) J_{0}\left(\frac{4 i a}{\left(1-\rho^{2}\right) E / 2 N_{0}} \sqrt{\mu}\right)
\end{aligned}
$$

where $J_{0}$ is the zeroth-order Bessel function of the first kind, and $i=\sqrt{-1}$.

However, $A=\rho \sqrt{\left(\left|\tilde{h}_{1}\right|^{2}+\left|h_{2}\right|^{2}\right) E / 2 N_{0}}$ is Rayleigh distributed with four degrees of freedom, and pdf

$$
P_{A}(a)=\frac{8 a^{3}}{\rho^{4}\left(E / N_{0}\right)^{2}} \exp \left(-2 a^{2} /\left(\rho^{2} E / N_{0}\right)\right)
$$

Integrating the product of (26) and (27) over the variable $a$ from 0 to $\infty$ leads to the following density probability for $\mu$ [3]

$$
p_{\mu}(\mu)=\lambda_{e}^{V \mu}{ }_{1} F_{1}(2,1 ; \eta \mu)
$$

where we have introduced the variables $\eta=2 \rho^{2} /((1-$ $\left.\left.\rho^{2}\right) E / N_{0}\right), \lambda=2\left(1-\rho^{2}\right) N_{0} / E, \nu=2 /\left(\left(1-\rho^{2}\right)\left(E / N_{0}\right)\right)$, and ${ }_{1} F_{1}(\alpha, \beta ; x)$ is the confluent hypergeometric function. Finally, by noticing [4] that ${ }_{1} F_{1}(2,1 ;-x)=(1-x) e^{-x},(28)$ can be written as

$$
p_{\mu}(\mu)=\lambda_{e}^{-(\nu-\eta) \mu}(1+\eta \mu)
$$

Averaging $Q(\sqrt{2 \mu})$ over this distribution yields

$$
\begin{aligned}
P_{e}= & \left(1-\rho^{2}\right)\left[\frac{1}{2}\left(1-\frac{1}{\sqrt{1+\frac{2}{E / N_{0}}}}\right)\right] \\
& +\rho^{2}\left[\frac{1}{4}\left(1-\frac{1}{\sqrt{1+\frac{2}{E / N_{0}}}}\right)^{2}\left(2+\frac{1}{\sqrt{1+\frac{2}{E / N_{0}}}}\right)\right] .
\end{aligned}
$$

As expected, (30) reduces to (16) for the static case $(\rho=1)$, and it reduces to (20) for the uncorrelated case $(\rho=0)$.

\section{B. Performance of the DF Detector}

The performance of the DF detector is easily approximated in terms of the performance of the $\mathrm{ZF}$ linear detector. Let us express the average bit-error probability of the DF detector as $P_{e}=(1 / 2) P_{1}+(1 / 2) P_{2}$, where $P_{i}=\operatorname{Pr}\left[\hat{x}_{i} \neq x_{i}\right]$.

First, let us compute $P_{1}$. Comparing (5) and (8) to (12), we see that the first output $z_{1}$ of the WMF is identical to the first output $y_{1}$ of the ZF linear detector. Hence, the symbol error probability $P_{1}$ for $x_{1}$ is given by (30).

An exact expression for $P_{2}$ has not been found. Instead, we will derive a lower bound on $P_{2}$ by assuming that the decision $\hat{x}_{1}$ is always correct. Under this assumption, the subtraction in

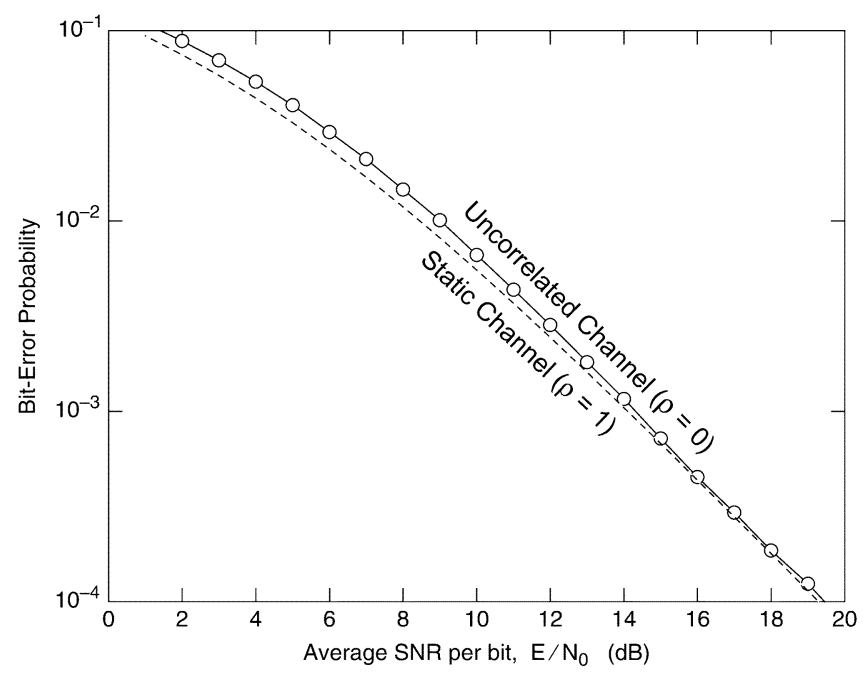

Fig. 1. Performance of the ML detector.

(9) cancels the crosstalk perfectly, leaving a difference given by

$$
D=\sqrt{\left|\tilde{h}_{1}\right|^{2}+\left|h_{2}\right|^{2}} x_{2}+n_{2}
$$

We recognize this as having precisely the same form as (14), the ideal two-fold diversity case. Hence, it follows immediately that $P_{2}$ is given by (16). Combining, we can bound the performance of the DF detector by

$$
\begin{aligned}
P_{e}> & \frac{1-\rho^{2}}{2}\left[\frac{1}{2}\left(1-\frac{1}{\sqrt{1+\frac{2}{E / N_{0}}}}\right)\right]+\frac{1+\rho^{2}}{2} \\
\times & {\left[\frac{1}{4}\left(1-\frac{1}{\sqrt{1+\frac{2}{E / N_{0}}}}\right)^{2}\left(2+\frac{1}{\sqrt{1+\frac{2}{E / N_{0}}}}\right)\right] . }
\end{aligned}
$$

\section{NUMERICAL RESULTS}

In this section, we compare the bit-error probability performance for the different detectors proposed in Section III.

It is difficult to analyze the performance of the ML detector when the channel is not static. Therefore, we rely on computer simulations instead. In Fig. 1, we present bit-error probability results for the ML detector for the uncorrelated case $(\rho=0)$ and the static channel $(\rho=1)$, the latter curve computed using (16). There is very little degradation due to the time-varying channel. Even when the channel varies so rapidly that the correlation is zero, the ML detector is able to perform almost as well as for the static channel.

The performance of the $\mathrm{ZF}$ linear detector is given in exact form by (30), and its behavior is shown in Fig. 2 for different values of $\rho$. We see that the performance depends strongly on the channel correlation characteristics. If the correlation is too small, the ZF linear detector will actually perform worse than a 


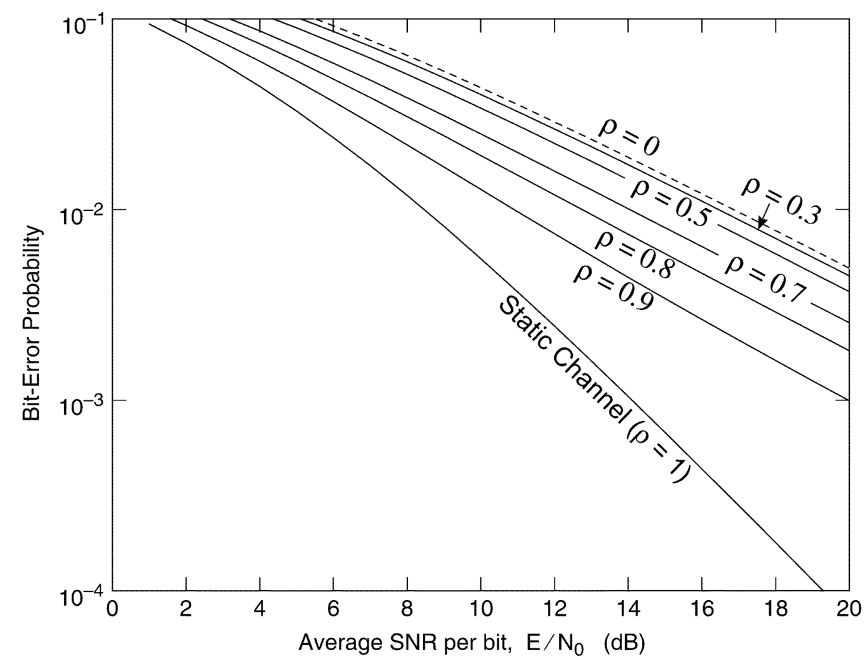

Fig. 2. Performance of the ZF detector.

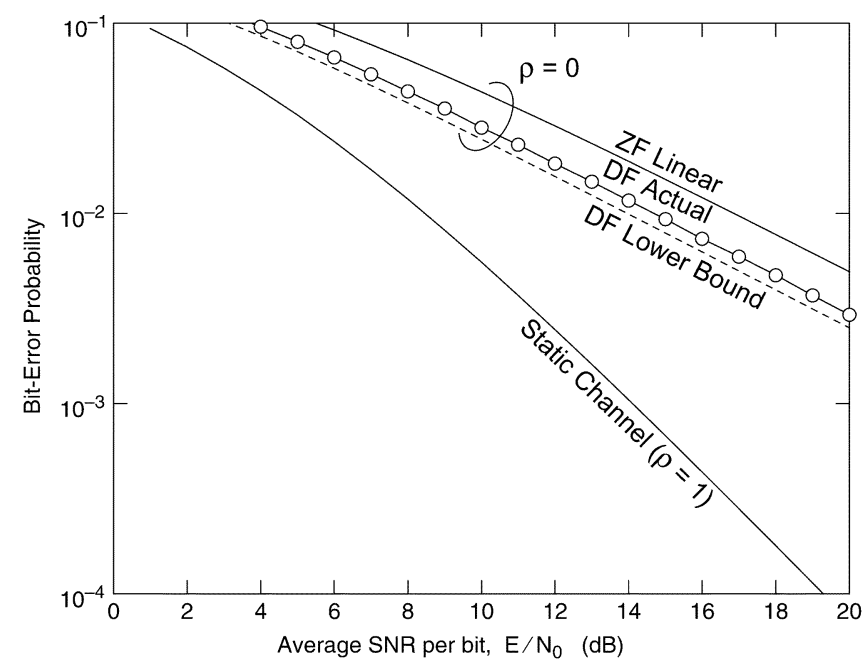

Fig. 3. Performance of the DF detector.

system with no diversity. Numerical calculations reveal that the ZF linear detector outperforms a receiver without diversity only when $\rho$ is approximately greater than 0.75 .

The performance of the DF detector is shown in Fig. 3, where Monte Carlo simulations of bit-error probability are compared with the lower bound of (32). The simulation results are more accurate because they account for the effect of occasionally feeding back erroneous decisions. The small gap between the lower bound and the simulated performance implies that the DF detector suffers a small penalty due to error propagation.

In Fig. 4, we compare the performance of all three detectors for three cases: $\rho=0, \rho=0.9$, and the static case $(\rho=1)$. When the channel varies with time, the ML detector is far superior to the other detectors. The ML detector suffers less than $1 \mathrm{~dB}$ of degradation when $\rho=0$ as compared to a static channel. Even when the correlation is as high as $\rho=0.9$, the ML detector significantly outperforms the others. When $P_{e}=10^{-2}$ and $\rho=0.9$, the ZF and DF detectors suffer a penalty of 2.5 and $1.5 \mathrm{~dB}$, respectively, compared to those in the static case. At $P_{e}=10^{-3}$ and $\rho=0.9$, the penalties grow to 4 and $6 \mathrm{~dB}$, respectively.

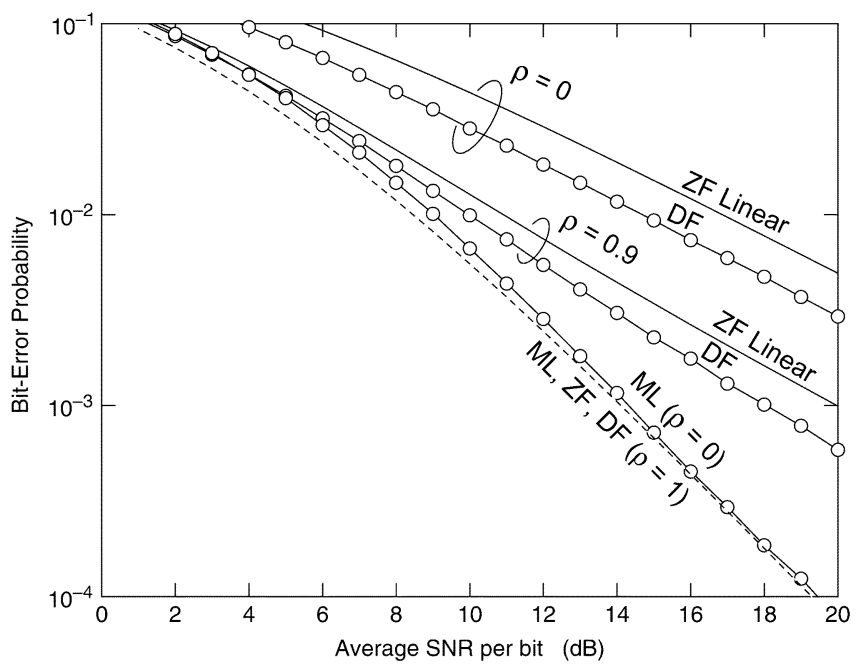

Fig. 4. Performance comparison for the different detectors.

\section{CONCLUSION}

We proposed three strategies for detecting an Alamouti transmit-diversity scheme when the channel is time-varying: the ML, DF, and linear detectors. Through analysis and simulation, we assessed their performance over time-varying Rayleigh fading channels as characterized by the channel correlation coefficient $\rho$. The ML detector significantly outperforms the DF and ZF detectors when the channel varies rapidly and $\rho$ is small. Only the ML detector is able to attain a diversity order of two. However, the ML detector can be significantly more complex, especially when higher-order alphabets are considered. The DF and ZF linear detectors are thus attractive, especially when the channel varies slowly.

\section{ACKNOWLEDGMENT}

The authors would like to thank G. H. Meyer for his mathematics advice and references.

\section{REFERENCES}

[1] S. M. Alamouti, "A simple transmit diversity technique for wireless communications," IEEE J. Select Areas Commun., vol. 16, pp. 1451-1458, Oct. 1998.

[2] J. G. Proakis, Digital Communications, 4th ed. New York: McGrawHill, 2001, pp. 824-825.

[3] I. S. Gradshtenyn, I. M. Ryzhik, and A. Jeffrey, Table of Integrals, Series, and Products, 5th ed. London, U.K.: Academic Press, 1997, p. 737.

[4] L. C. Andrews, Special Functions for Engineers and Applied Mathematics. New York: Macmillan, 1995, p. 313.

[5] G. L. Stüber, Principles of Mobile Communication, 2nd ed. Norwell, MA: Kluwer, Nov. 2000.

[6] P. W. Wolniansky, G. J. Foschini, G. D. Golden, and R. A. Valenzuela, "V-BLAST: An architecture for realizing very high data rates over the rich-scattering wireless channel," in Proc. URSI Int. Symp. Signals, Systems, and Electronics, Pisa, Italy, Sept. 29, 1998, pp. 295-300.

[7] W. C. Jakes, Microwave Mobile Communications. New York: IEEE Press, 1974.

[8] G. J. Foschini and M. J. Gans, "On limits of wireless communications in a fading environment when using multiple antennas," Wireless Personal Commun., vol. 6, pp. 311-335, Mar. 1998.

[9] A. Duel-Hallen, "Decorrelating decision-feedback multiuser detector for synchronous code-division multiple access channel," IEEE Trans. Commun., vol. 41, pp. 285-290, Feb. 1993. 